\title{
SUZAKU DISCOVERY OF THE STRONG RADIATIVE RECOMBINATION CONTINUUM OF IRON FROM THE SUPERNOVA REMNANT W49B
}

\section{$\operatorname{AUTHOR}(\mathrm{S}):$}

Ozawa, M.; Koyama, K.; Yamaguchi, H.; Masai, K.; Tamagawa, $\mathrm{T}$.

\section{CITATION:}

Ozawa, M....[et al]. SUZAKU DISCOVERY OF THE STRONG RADIATIVE RECOMBINATION CONTINUUM OF IRON FROM THE SUPERNOVA REMNANT W49B. The Astrophysical Journal 2009, 706(1): L71-L75

\section{ISSUE DATE:}

2009-11-30

URL:

http://hdl.handle.net/2433/139435

RIGHT:

(c) 2009 The American Astronomical Society 


\title{
SUZAKU DISCOVERY OF THE STRONG RADIATIVE RECOMBINATION CONTINUUM OF IRON FROM THE SUPERNOVA REMNANT W49B
}

\author{
M. Ozawa ${ }^{1}$, K. Koyama ${ }^{1}$, H. Yamaguchi ${ }^{2}$, K. Masai $^{3}$, and T. Tamagawa ${ }^{2}$ \\ ${ }^{1}$ Department of Physics, Kyoto University, Kitashirakawa-oiwake-cho, Sakyo-ku, Kyoto 606-8502, Japan; midori@ cr.scphys.kyoto-u.ac.jp \\ ${ }^{2}$ RIKEN (The Institute of Physical and Chemical Research), 2-1 Hirosawa, Wako, Saitama 351-0198, Japan \\ ${ }^{3}$ Department of Physics, Tokyo Metropolitan University, 1-1 Minami-Osawa, Hachioji, Tokyo 192-0397, Japan \\ Received 2009 September 10; accepted 2009 October 16; published 2009 October 30
}

\begin{abstract}
We present a hard X-ray spectrum of unprecedented quality of the Galactic supernova remnant (SNR) W49B obtained with the Suzaku satellite. The spectrum exhibits an unusual structure consisting of a saw-edged bump above $8 \mathrm{keV}$. This bump cannot be explained by any combination of high-temperature plasmas in ionization equilibrium. We firmly conclude that this bump is caused by the strong radiative recombination continuum (RRC) of iron, detected for the first time in a SNR. The electron temperature derived from the bremsstrahlung continuum shape and the slope of the RRC is $\sim 1.5 \mathrm{keV}$. On the other hand, the ionization temperature derived from the observed intensity ratios between the RRC and $\mathrm{K} \alpha$ lines of iron is $\sim 2.7 \mathrm{keV}$. These results indicate that the plasma is in a highly overionized state. Volume emission measures independently determined from the fluxes of the thermal and RRC components are consistent with each other, suggesting the same origin of these components.
\end{abstract}

Key words: ISM: individual (W49B) - radiation mechanisms: thermal - supernova remnants - X-rays: ISM

\section{INTRODUCTION}

W49B (G43.3-0.2) is a Galactic supernova remnant (SNR) with strong X-ray line emissions from highly ionized atoms. It exhibits centrally filled X-rays inside a bright radio shell with a radius of 100 arcsec (Pye et al. 1984). The distance to W49B remains uncertain. It was estimated to lie at a distance of $\sim 8 \mathrm{kpc}$ (Radhakrishnan et al. 1972; Moffett \& Reynolds 1994). Using the spectral distribution of $\mathrm{HI}$ absorption, however, Brogan \& Troland (2001) found no clear evidence that W49B is closer to the Sun than W49A, which is located at a distance of $\sim 11.4 \mathrm{kpc}$ (Gwinn et al. 1992). This may indicate a possible association of W49B with the star-forming region W49A. The near-infrared narrowband images indicate a barrel-shaped structure with coaxial rings, which is suggestive of bipolar wind structures surrounding massive stars (Keohane et al. 2007). They also showed an X-ray image from Chandra, which has a jet-like structure along the axis of the barrel. They interpreted these findings as evidence that W49B had exploded inside a windblown bubble in a dense molecular cloud.

Using ASCA data, Hwang et al. (2000) showed that broadband modeling of the remnant's spectrum required two thermal components $(0.2 \mathrm{keV}$ and $2 \mathrm{keV})$ and significant overabundances of $\mathrm{Si}, \mathrm{S}, \mathrm{Ar}, \mathrm{Ca}, \mathrm{Fe}$, and $\mathrm{Ni}$. They confirmed that most of the X-ray emitting plasma was nearly in collisional ionization equilibrium (CIE). They also found evidence for $\mathrm{Cr}$ and $\mathrm{Mn} \mathrm{K} \alpha$ emission.

Kawasaki et al. (2005) claimed that there was a presence of "overionized" plasma in W49B through the analysis of ASCA $2.75-6.0 \mathrm{keV}$ spectrum. They measured intensity ratios of the $\mathrm{H}$-like $\mathrm{K} \alpha$ (hereafter $\mathrm{Ly} \alpha$ ) to He-like $\mathrm{K} \alpha(\mathrm{He} \alpha)$ lines of $\mathrm{Ar}$ and $\mathrm{Ca}$ to obtain the ionization temperature $\left(k T_{z}\right)$, and found that $k T_{z}(\sim 2.5 \mathrm{keV})$ is significantly higher than the electron temperature $\left(k T_{\mathrm{e}}\right)$ determined from the bremsstrahlung continuum shape $(\sim 1.8 \mathrm{keV})$. Miceli et al. (2006) adopted the same analysis procedure for the XMM-Newton spectrum of the central region but found no evidence for the overionized state.

In this Letter, we report the firm evidence for overionized plasma in W49B using data from the X-ray Imaging Spectrom- eters (XISs; Koyama et al. 2007) onboard the Suzaku satellite (Mitsuda et al. 2007).

\section{OBSERVATION AND DATA REDUCTION}

We observed W49B with Suzaku on 2009 March 29 and 31 (observation IDs 503084010 and 504035010, respectively). Because the pointing positions and rotation angles do not differ significantly, we merged these two data sets. The XIS consists of four X-ray CCD camera systems placed on the focal planes of four X-ray telescopes (Serlemitsos et al. 2007). Three of them were operated in the normal full-frame clocking mode with a spaced-row charge injection technique (Uchiyama et al. 2009) during our observations. Two are front-illuminated (FI) CCDs and the other is a back-illuminated (BI) CCD. Data were cleaned using processing version 2.2.11.24. We used HEASOFT version 6.5.1 for data reduction and XSPEC version 11.3.2 for spectral analysis. After screening with the standard criteria, ${ }^{4}$ the net integration time was $\sim 113 \mathrm{ks}$.

\section{ANALYSIS}

\subsection{Construction of the Spectrum}

Figure 1 shows the vignetting-corrected XIS image in 1.5$7 \mathrm{keV}$, the energy band including the major lines of K-shell emissions from $\mathrm{Si}, \mathrm{S}, \mathrm{Ar}, \mathrm{Ca}$, and $\mathrm{Fe}$. We extracted the source spectrum from a circular region with a radius of 2.5 arcmin and the background spectrum from an annulus surrounding the source with inner and outer radii of 5 and 8 arcmin, respectively. Non-X-ray background (NXB) spectra constructed with the xisnxbgen software were subtracted from both the source and background data. After correcting the vignetting and accumulation area, the source spectrum was made by subtracting the background spectrum. The spectra of the two FI sensors were merged to improve the statistics because their response functions were almost identical. Figure 2 shows the merged FI spectrum. We can see several prominent lines of K-shell emission from $\mathrm{He}-$ and H-like ions.

\footnotetext{
4 http://heasarc.nasa.gov/docs/suzaku/processing/criteria_xis.html
} 


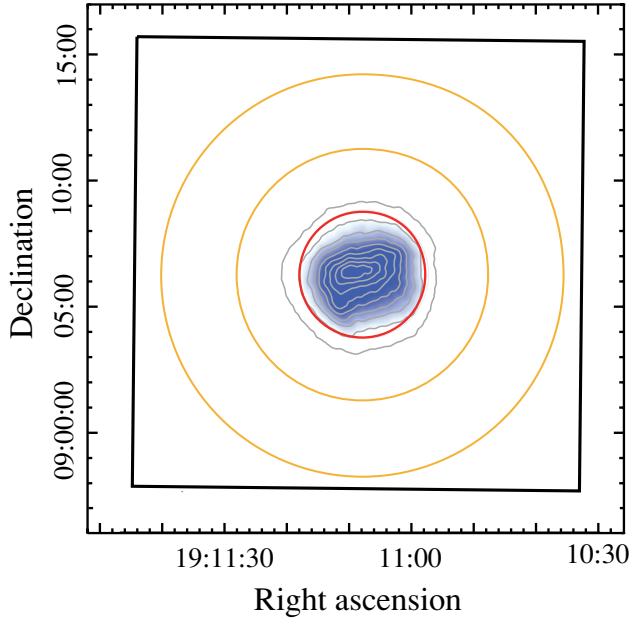

Figure 1. Vignetting-corrected XIS image of W49B in the $1.5-7 \mathrm{keV}$ band shown on a linear intensity scale. Data from the three active XISs are combined. Gray contours indicate every $10 \%$ intensity level relative to the peak surface brightness. The red circle and orange annulus indicate the source and background regions, respectively. The XIS field of view is shown by the black square.

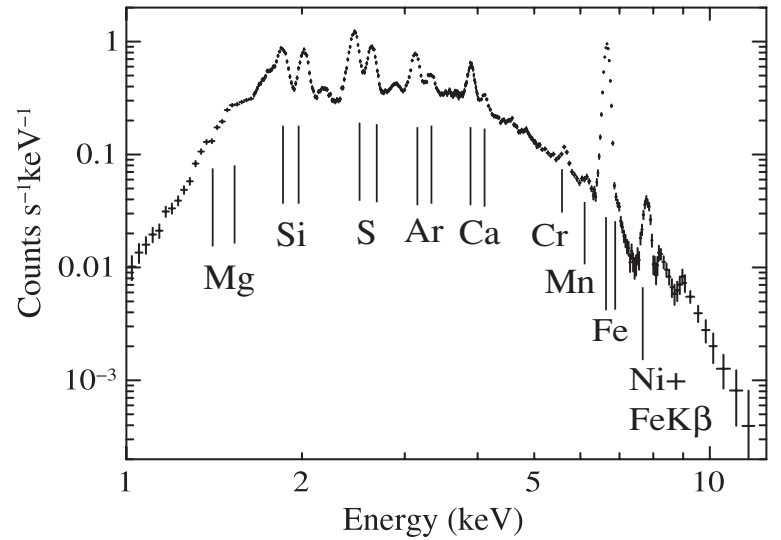

Figure 2. Background-subtracted XIS FI spectrum. The energies of the prominent emission lines from specific elements are labeled.

\subsection{APEC Model Fit in the Hard X-ray Band}

As shown in Figure 2, the wide-band spectrum includes too many emission lines and possibly other complicated structures. For clean analyses and discussion, we focus on the 5-12 keV energy band, where emission lines and other structures mainly originate from Fe. We used only the FI data because the NXB count rate of the BI data is much larger than the source rate in this energy band.

We first fitted the spectrum with a VAPEC (CIE plasma) model (Smith et al. 2001). The Fe and Ni abundances (hereafter $Z_{\mathrm{Fe}}$ and $Z_{\mathrm{Ni}}$ ) normalized by the number fraction of the solar photosphere (Anders \& Grevesse 1989) were free parameters. Since the $\mathrm{Cr}$ and $\mathrm{Mn} \mathrm{K} \alpha$ emission lines are not included in the VAPEC model, we added these lines with Gaussian functions. We fixed the interstellar extinction to a hydrogen column density of $5 \times 10^{22} \mathrm{~cm}^{-2}$ with the solar elemental abundances, following Hwang et al. (2000). To fine tune the energy scale, an offset was added as a free parameter and found to be $-4.7 \mathrm{eV}$, which was within the allowable range of the calibration uncertainties. We added this offset for further spectral fitting. Figure 3(a) shows the fitting result of the one-VAPEC model, with a temperature of $1.64 \mathrm{keV}$. The model exhibited significant excess around the
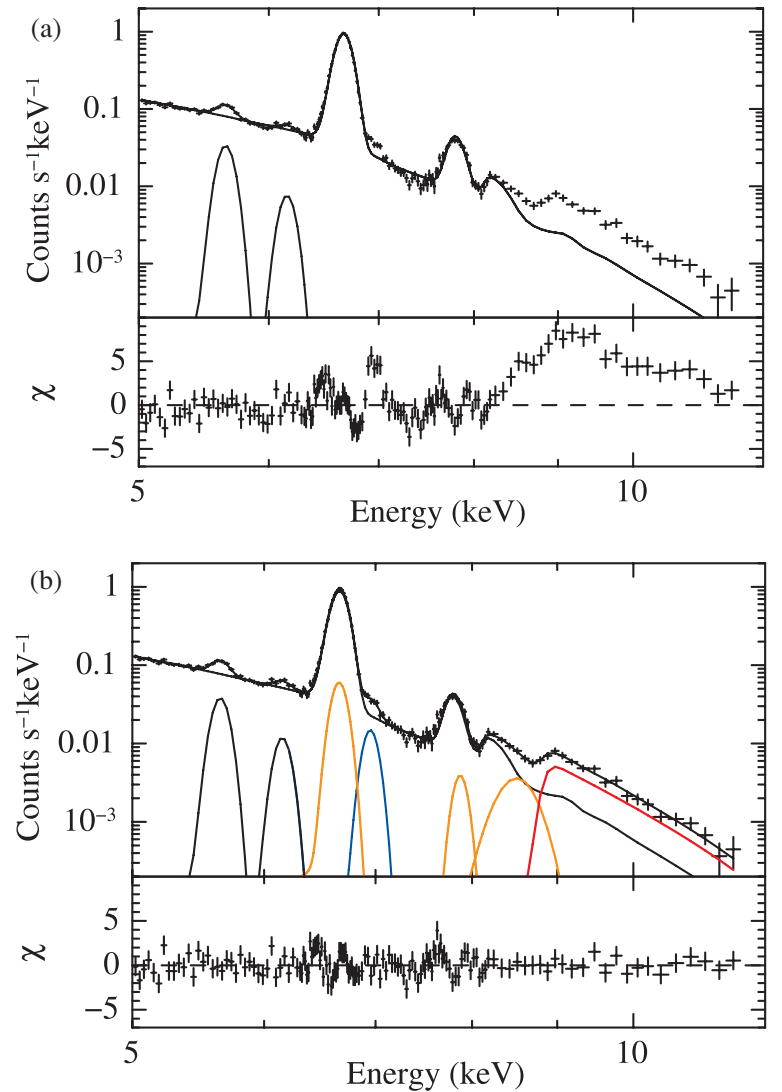

Figure 3. (a) XIS spectrum in the 5-12 keV band. The best-fit VAPEC model and additional $\mathrm{K} \alpha$ lines of $\mathrm{Cr}$ and $\mathrm{Mn}$ with Gaussian functions are shown by solid lines. The lower panel shows the residual from the best-fit model. (b) Same spectrum as (a), but with the radiative recombination continuum (red), recombination lines (orange), and a Ly $\alpha$ line (blue) of Fe.

Fe Ly $\alpha$ line and above $8 \mathrm{keV}$, and hence was rejected with a large $\chi^{2} /$ degree of freedom (dof) of 1051/138.

We then tried a two-VAPEC model, assuming equal abundances between the two components. Fe Ly $\alpha$ was successfully reproduced, but the temperature of one component was unreasonably high $(\sim 70 \mathrm{keV})$, and a large residual above $8 \mathrm{keV}$ remained with an unacceptable $\chi^{2} /$ dof of 562/136. Adding a further plasma or power-law component did not improve the fitting any more. In every case, the large bump above $8 \mathrm{keV}$ was present.

To examine whether this bump is real or artificial, we checked the light curves of the source and background regions in the 8.5$10 \mathrm{keV}$ band. They showed almost constant fluxes, indicating no flare-like event had occurred during the observations. In addition, we detected the bump in both FI spectra (XIS 0 and XIS 3) and even in the BI spectrum. Thus, the bump is a real structure.

The edge energy of the bump $(\sim 9 \mathrm{keV})$ corresponds to the electron binding energy of Fe. This suggests that the saw-edged bump is likely due to a radiative recombination continuum (RRC): X-ray emissions due to the free-bound transition of electrons. Hereafter, we call the RRC accompanied by the recombination of $\mathrm{H}$-like ions into the ground state of He-like ions the He-RRC, and that of fully ionized ions into the ground state of H-like ions the H-RRC.

\subsection{Recombination Continuum and Lines}

Both the line-like excess around $\mathrm{Fe}$ Ly $\alpha$ and the bump above $8 \mathrm{keV}$ are suggestive of the overionized state because there 
Table 1

Best-Fit Spectral Parameters

\begin{tabular}{|c|c|c|c|c|}
\hline CIE (VAPEC) & $k T_{\mathrm{e}}(\mathrm{keV})$ & $Z_{\mathrm{Fe}}($ solar $)$ & $Z_{\mathrm{Ni}}($ solar $)$ & Normalization $^{\mathrm{a}}$ \\
\hline & $1.52(1.50-1.53)$ & $4.44(4.36-4.53)^{b}$ & $10.9(9.14-12.7)^{b}$ & $1.61(1.60-1.62)$ \\
\hline \multirow[t]{7}{*}{ Line } & & Center energy (keV) & Line width (keV) & Normalization $^{\mathrm{c}}$ \\
\hline & $\mathrm{Cr} \mathrm{K} \alpha$ & $5.655(5.646-5.663)$ & 0 (fixed) & $2.85(2.59-3.11)$ \\
\hline & $\operatorname{Mn~K} \alpha$ & $6.162(6.142-6.183)$ & 0 (fixed) & $0.957(0.750-1.16)$ \\
\hline & $\mathrm{Fe} \operatorname{He} \alpha_{\mathrm{rec}}^{\mathrm{d}}$ & $6.666\left(\right.$ fixed $\left.^{\mathrm{e}}\right)$ & 0 (fixed) & $5.30(4.20-6.39)$ \\
\hline & $\operatorname{Fe} \operatorname{Ly} \alpha$ & $6.961(6.946-6.971)$ & 0 (fixed) & $1.46(1.27-1.65)$ \\
\hline & $\mathrm{Fe} \operatorname{He} \beta_{\mathrm{rec}} \mathrm{d}^{\mathrm{d}}$ & $7.880\left(\right.$ fixed $\left.^{\mathrm{e}}\right)$ & 0 (fixed) & $0.593(0.338-0.847)$ \\
\hline & Fe He $\gamma-\infty_{\text {rec }}{ }^{d}$ & $8.538(8.492-8.585)$ & $0.200(0.163-0.246)$ & $2.12(1.81-2.43)$ \\
\hline \multirow[t]{3}{*}{ RRC } & & Edge energy (keV) & $k T_{\mathrm{e}}(\mathrm{keV})$ & Normalization $^{\mathrm{c}}$ \\
\hline & $\mathrm{Fe} \mathrm{He}-\mathrm{RRC}$ & 8.830 (fixed) & $1.43(1.30-1.59)^{\mathrm{f}}$ & $11.2(10.4-11.9)$ \\
\hline & Fe H-RRC & 9.194 (fixed) & $1.43(1.30-1.59)^{\mathrm{f}}$ & $0.257(0.00-1.04)$ \\
\hline
\end{tabular}

Notes. The uncertainties in the parentheses are the $90 \%$ confidence range.

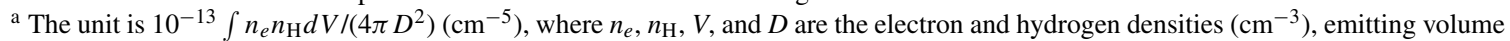
$\left(\mathrm{cm}^{3}\right)$, and distance to the source $(\mathrm{cm})$, respectively.

b These values should be modified in the case of the overionized plasma. See Section 4.3 for details.

c The unit is $10^{-5} \mathrm{~cm}^{-2} \mathrm{~s}^{-1}$.

${ }^{\mathrm{d}}$ Lines emitted by a recombination process.

${ }^{\mathrm{e}}$ Fixed at the experimental values (Wargelin et al. 2005).

${ }^{\mathrm{f}}$ We assumed the same $k T_{\mathrm{e}}$.

should be a greater fraction of H-like ions in the overionized state than in the CIE. This excessive number of H-like ions causes strong He-RRC and Fe Ly $\alpha$ emissions. However, no current plasma code can be applied to the overionized plasma. $\mathrm{We}$, therefore, introduce the $\mathrm{Fe} \operatorname{Ly} \alpha$ line and recombination structures consisting of the RRC and several emission lines below the K-edge energy of the RRC ( $\left.E_{\text {edge }}\right)$, in addition to the one-VAPEC model. We consider both He-RRC and H-RRC for a consistency check (see Section 4.2).

We assume that the RRC is expressed as

$$
\frac{d N}{d E} \propto \exp \left(-\frac{E-E_{\text {edge }}}{k T_{\mathrm{e}}}\right), \text { for } E \geqslant E_{\text {edge }},
$$

where $k T_{\mathrm{e}}$ is the electron temperature of the relevant recombining plasma. This formula gives a good approximation when the electron temperature is much lower than the K-edge energy $\left(k T_{\mathrm{e}} \ll E_{\text {edge }}\right.$; e.g., Smith \& Brickhouse 2002). In the case of W49B, $k T_{\mathrm{e}}$ is $\sim 1.5 \mathrm{keV}$, as we confirm later, while $E_{\text {edge }}$ of Fe is $\sim 9 \mathrm{keV}$. Thus, we can safely adopt this formula.

The recombination lines originate from cascade decays of electrons that are captured into the excited levels of ions by the free-bound transition. We designate the cascade lines of Helike $\mathrm{Fe}$ as $\mathrm{He} \alpha_{\text {rec }}$ (the principle quantum number $n=2 \rightarrow 1$ ) and $\mathrm{He} \beta_{\text {rec }}(3 \rightarrow 1)$. For other lines from higher levels $(n \geqslant 4 \rightarrow 1)$, we combine them into one broad Gaussian function $\left(\mathrm{He} \gamma-\infty_{\text {rec }}\right)$. The line widths and center energies of $\mathrm{He} \alpha_{\text {rec }}$ and $\mathrm{He} \beta_{\text {rec }}$ are fixed at zero and the experimental values for a charge exchange process, respectively (Wargelin et al. 2005). On the other hand, those of $\mathrm{He} \gamma-\infty_{\text {rec }}$ are allowed to vary freely. The fluxes of all these lines are free parameters.

The resultant best-fit parameters and models are given in Table 1 and Figure 3(b), respectively. With this model, $\chi^{2} /$ dof is greatly improved to $193 / 128$, although this is still unacceptable in a purely statistical sense. We can see significant data excesses at the lower energy sides of the $\mathrm{He} \alpha$ lines of $\mathrm{Fe}(\sim 6.5 \mathrm{keV})$ and $\mathrm{Ni}(\sim 7.6 \mathrm{keV})$. The former is likely due to the incomplete response function. We should note that $\mathrm{Fe} \mathrm{He} \alpha$ statics are superior to those for any other objects observed with Suzaku, and no significant residual has appeared so far. If we observe carefully, we find a similar feature in the $\mathrm{Mn} \mathrm{K} \alpha$ line from the onboard calibration source. The latter would be partially due to the same reason given above, but is mainly due to lack of satellite lines of the Be-like and lower ionization states ${ }^{5}$ of $\mathrm{Ni}$ in the VAPEC model. If we ignore the $6.4-6.6 \mathrm{keV}$ and 7.6$7.7 \mathrm{keV}$ band to escape these effects, an acceptable $\chi^{2} / \mathrm{dof}$ of $118 / 107$ is obtained.

\section{RESULTS AND DISCUSSION}

We have found, for the first time, the strong He-RRC of Fe from W49B. Yamaguchi et al. (2009) recently discovered the $\mathrm{H}-\mathrm{RRC}$ of Si and S from a middle-aged SNR, IC 443, and hence this is the second discovery of a clear RRC from an SNR. We also discovered RRC-accompanied recombination lines, which may provide good diagnostics for the overionized plasma. We review a quantitative verification of our spectral analysis and discuss the implications of the results.

\subsection{Contribution of the Recombination Lines}

We discuss the validity of the best-fit fluxes of the RRC and recombination lines in Table 1. The recombination cross section of the H-like ions into a level of $n$ is approximately given as shown below (e.g., Nakayama \& Masai 2001):

$$
\sigma_{n} \propto \frac{1}{n^{3}}\left(\frac{3}{2} \frac{k T_{\mathrm{e}}}{E_{\text {edge }}}+\frac{1}{n^{2}}\right)^{-1} .
$$

We apply this approximation for the He-like ions, but $\sigma_{1}$ must be reduced by half because one electron is already at the ground state. In principle, the recombination line flux can be estimated by the branching ratio to various levels, but these processes are very complicated. We, therefore, base our discussion only on a simple picture.

We compare the predicted capture and observed transition rates normalized with the $n=1$ value. We can estimate the capture rates using Equation (2) as $\sigma_{2} / \sigma_{1}=0.62, \sigma_{3} / \sigma_{1}=0.25$,

\footnotetext{
5 As for Fe, such satellite lines are included in the VAPEC model.
} 


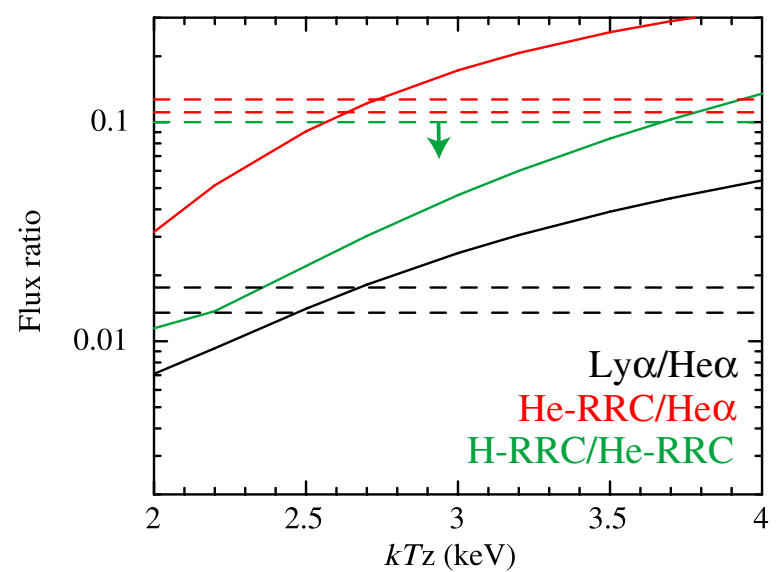

Figure 4. Predicted emissivity ratios of $\mathrm{Ly} \alpha / \mathrm{He} \alpha$ (black), $\mathrm{He}-\mathrm{RRC} / \mathrm{He} \alpha$ (red), and $\mathrm{H}-\mathrm{RRC} / \mathrm{He}-\mathrm{RRC}$ (green) of $\mathrm{Fe}$ as a function of the ionization temperature $\left(k T_{z}\right)$ for an electron temperature of $1.5 \mathrm{keV}$ (Masai 1994). The horizontal dashed lines represent $90 \%$ errors of the observed values.

and $\left(\sigma_{4}+\sigma_{5}+\cdots\right) / \sigma_{1}=0.34$ for $k T_{\mathrm{e}}=1.5 \mathrm{KeV}$ and $E_{\text {edge }}=$ $8.83 \mathrm{KeV}$. The observed flux rates are $\mathrm{He} \alpha_{\mathrm{rec}} / \mathrm{He}-\mathrm{RRC}=0.47$ $( \pm 0.10), \mathrm{He} \beta_{\text {rec }} / \mathrm{He}-\mathrm{RRC}=0.053( \pm 0.023)$, and $\mathrm{He} \gamma-\infty_{\text {rec }} /$ He-RRC $=0.19( \pm 0.03) .{ }^{6}$ By taking the fractions between the observed and predicted rates, we obtain $76( \pm 16) \%, 21$ $( \pm 9) \%$, and $56( \pm 9) \%$ for the $\mathrm{He} \alpha_{\text {rec }}, \mathrm{He} \beta_{\text {rec }}$, and $\mathrm{He} \gamma-\infty_{\text {rec }}$ lines, respectively.

These fractions for the $\mathrm{He} \beta_{\text {rec }}$ and $\mathrm{He} \gamma-\infty_{\text {rec }}$ lines may be conceivable, because one electron at $n=1$ suppresses direct transitions from excited levels $(n \geqslant 3 \rightarrow 1)$ for the He-like ions. The fraction of $\operatorname{He} \alpha_{\text {rec }}$ is slightly smaller than expected because there should be a significant contribution of the cascade decay electrons from higher levels $(n \geqslant 3 \rightarrow 2 \rightarrow 1)$. The real He $\alpha_{\text {rec }}$ flux may be somewhat larger due to the uncertainty of the response function, but the contribution of the $\mathrm{He} \alpha_{\text {rec }}$ flux relative to the total $\mathrm{He} \alpha$ is only $\lesssim 10 \%$ and does not affect results and following discussion.

\subsection{Electron and Ionization Temperatures}

The electron temperatures determined by the bremsstrahlung continuum shape and the RRC slope are 1.52 (1.50-1.53) keV and 1.43 (1.30-1.59) keV, respectively. These consistent results indicate a common origin of these emissions.

The ionization temperatures directly reflect the ion fractions and hence can be determined as shown below. From the best-fit model in Table 1, the flux ratios of $\mathrm{Ly} \alpha / \mathrm{He} \alpha, \mathrm{He}-\mathrm{RRC} / \mathrm{He} \alpha$, and $\mathrm{H}-\mathrm{RRC} / \mathrm{He}-\mathrm{RRC}$ are given as 0.016 (0.014-0.018), 0.12 (0.11$0.13)$, and $0.023(\leqslant 0.10)$. In Figure 4, we compare these values with the modeled emissivity ratios derived from the radiation code of Masai (1994) for a plasma of $k T_{\mathrm{e}}=1.5 \mathrm{keV}$. We obtain $k T_{z}=2.58(2.46-2.68) \mathrm{keV}, 2.68(2.63-2.73) \mathrm{keV}$, and 2.55 $(\leqslant 3.65) \mathrm{keV}$, respectively, for the above ratios. We also compare the $\mathrm{Ly} \alpha / \mathrm{He} \alpha$ ratio with that derived from the APEC code (Smith et al. 2001), although this code is valid only for a CIE state. We obtain $k T_{z}=2.46(2.39-2.54) \mathrm{keV}$, which is within the margin of error of the above results. The ionization temperatures $(\sim 2.7 \mathrm{keV})$ are significantly higher than the electron temperatures $(\sim 1.5 \mathrm{keV})$, indicating that the plasma is in a highly overionized state.

The first hint of overionized plasma in W49B was found by Kawasaki et al. (2005). Although they analyzed different

\footnotetext{
6 Throughout this Letter, all the errors in the parentheses are at $90 \%$
} confidence level. elements (Ar and $\mathrm{Ca}$ ) in different energy bands (2.75-6.0 keV), and derived $k T_{z}$ from the $\mathrm{Ly} \alpha / \mathrm{He} \alpha$ ratio using the CIE plasma code, their results $\left(k T_{z} \sim 2.5 \mathrm{keV}\right.$ and $\left.k T_{\mathrm{e}} \sim 1.8 \mathrm{keV}\right)$ are nearly consistent with ours. Our claim is more essential because it is based on clear detection of the recombination structures.

\subsection{Iron and Nickel Abundances}

The abundances listed in Table 1 are valid only for the CIE state and should be modified in the overionized case. Since no plasma code can be applied to the overionized plasma currently, we make possible modifications using an available APEC code.

The He $\alpha$ intensity is proportional to $Z \times \epsilon\left(k T_{\mathrm{e}}, k T_{z}\right)$, where $Z$ and $\epsilon\left(k T_{\mathrm{e}}, k T_{z}\right)$ are the abundance of the element (solar) and the total emissivity for the $\mathrm{He} \alpha$ for $k T_{\mathrm{e}}$ and $k T_{z}\left(\mathrm{~cm}^{3} \mathrm{~s}^{-1}\right)$, respectively. The emissivities of the $\mathrm{He}-, \mathrm{Li}-, \mathrm{Be}-$, and $\mathrm{B}-\mathrm{like}$ ions for $\mathrm{Fe}$ and the $\mathrm{He}$ - and $\mathrm{Li}$-like ions for $\mathrm{Ni}$ are modified by multiplying the ion-fraction ratio between $k T_{z}=2.7 \mathrm{keV}$ and $1.5 \mathrm{keV}$ (Mazzotta et al. 1998). The total emissivity is given by adding those in individual ionization states. Multiplying by $\epsilon(1.5 \mathrm{keV}, 1.5 \mathrm{keV}) / \epsilon(1.5 \mathrm{keV}, 2.7 \mathrm{keV})$, we obtain the real abundances in the overionized state as $Z_{\mathrm{Fe}} \sim 4.9$ solar and $Z_{\mathrm{Ni}} \sim 5.2$ solar. Both the elements are highly over abundant, indicating an ejecta origin of the plasma.

\subsection{Volume Emission Measure}

To check the consistency of the common origin of the bremsstrahlung and RRC emissions, we compare the volume emission measure (VEM). The VEM is given by $\int n_{e} n_{\mathrm{H}} d V /\left(4 \pi D^{2}\right)$, where $n_{e}, n_{\mathrm{H}}, V$, and $D$ are the electron and hydrogen densities $\left(\mathrm{cm}^{-3}\right)$, emitting volume $\left(\mathrm{cm}^{3}\right)$, and distance to the source $(\mathrm{cm})$, respectively.

The VEM of the VAPEC component $\left(\mathrm{VEM}_{\mathrm{VAPEC}}\right)$ is derived from Table 1 as $\mathrm{VEM}_{\mathrm{VAPEC}}=1.61(1.60-1.62) \times 10^{13} \mathrm{~cm}^{-5}$. On the other hand, the VEM of the RRC plasma $\left(\mathrm{VEM}_{\mathrm{RRC}}\right)$ is calculated from

$$
F_{\mathrm{RRC}}=\alpha_{1}\left(k T_{\mathrm{e}}\right) \times \frac{n_{\mathrm{Fe}}}{n_{\mathrm{H}}} \times \kappa_{\mathrm{H}-\mathrm{like}}\left(k T_{z}\right) \times \mathrm{VEM}_{\mathrm{RRC}},
$$

where $F_{\mathrm{RRC}}, \alpha_{1}\left(k T_{\mathrm{e}}\right), n_{\mathrm{Fe}}$, and $\kappa_{\mathrm{H}-\text { like }}\left(k T_{z}\right)$ are the flux of the He-RRC $\left(\mathrm{cm}^{-2} \mathrm{~s}^{-1}\right)$, the K-shell recombination rate coefficient for $k T_{\mathrm{e}}\left(\mathrm{cm}^{3} \mathrm{~s}^{-1}\right)$, the number density of $\mathrm{Fe}\left(\mathrm{cm}^{-3}\right)$, and the ion fraction of $\mathrm{H}$-like $\mathrm{Fe}$ for $k T_{z}$, respectively. According to Badnell (2006), the total radiative recombination rate of $\mathrm{He}$ like $\mathrm{Fe}$ at $k T_{\mathrm{e}}=1.5 \mathrm{keV}$ is given as $\sim 3.9 \times 10^{-12} \mathrm{~cm}^{3} \mathrm{~s}^{-1}$. The recombination rate into the ground state is given using Equation (2) as $\sigma_{1} /\left(\sigma_{1}+\sigma_{2}+\cdots\right) \sim 0.45$. The value of $n_{\mathrm{Fe}} / n_{\mathrm{H}}$ is calculated using $Z_{\mathrm{Fe}}$ in Section 4.3 and the number density of the solar photosphere (Anders \& Grevesse 1989). Using the observed He-RRC flux, we obtain ${ }^{7} \mathrm{VEM}_{\mathrm{RRC}}=0.81(0.58-1.60) \times$ $10^{13} \mathrm{~cm}^{-5}$. The two independent estimations of VEM give consistent results, supporting the same origin of the overionized plasma in W49B.

The origin of the overionized plasma in SNRs is an open question, and beyond the scope of this Letter. We simply note the possibility of the cooling of electrons via thermal conduction (Kawasaki et al.2005) or a more drastic cooling caused when the blast wave breaks out of some ambient matter into the rarefied interstellar medium (Yamaguchi et al. 2009). If the latter is the case, a massive progenitor that had blown a stellar wind is likely to be favored.

\footnotetext{
7 Here, we consider that the $k T_{z}$ error is $2.4-2.8 \mathrm{keV}$. The large error of $V_{\text {VEM }}$ is due to this effect.
} 
The authors thank the anonymous referee for the valuable comments on our previous draft. We are grateful to Takeshi Tsuru, Hironori Matsumoto, Hideki Uchiyama, Asami Hayato, and the W49B team members for their constructive suggestions. We especially thank Masaomi Tanaka and Nozomu Tominaga for the dedicated support in giving us very helpful advice and improving the draft. M. Ozawa is a Research Fellow of the Japan Society for Promotion of Science (JSPS). This work is partially supported by the Grant-in-Aid for the Global COE Program "The Next Generation of Physics, Spun from Universality and Emergence," Challenging Exploratory Research (K.K.), and Young Scientists (H.Y.) from the Ministry of Education, Culture, Sports, Science and Technology (MEXT) of Japan.

\section{REFERENCES}

Anders, E., \& Grevesse, N. 1989, Geochim. Cosmochim. Acta, 53, 197 Badnell, N. R. 2006, ApJS, 167, 334

Brogan, C. L., \& Troland, T. H. 2001, ApJ, 550, 799

Gwinn, C. R., Moran, J. M., \& Reid, M. J. 1992, ApJ, 393, 149

Hwang, U., Petre, R., \& Hughes, J. P. 2000, ApJ, 532, 970
Kawasaki, M., Ozaki, M., Nagase, F., Inoue, H., \& Petre, R. 2005, ApJ, 631, 935

Keohane, J. W., Reach, W. T., Rho, J., \& Jarrett, T. H. 2007, ApJ, 654, 938

Koyama, K., et al. 2007, PASJ, 59, 23

Masai, K. 1994, ApJ, 437, 770

Mazzotta, P., Mazzitelli, G., Colafrancesco, S., \& Vittorio, N. 1998, A\&AS, 133, 403

Miceli, M., Decourchelle, A., Ballet, J., Bocchino, F., Hughes, J. P., Hwang, U., \& Petre, R. 2006, A\&A, 453, 567

Mitsuda, K., et al. 2007, PASJ, 59, 1

Moffett, D. A., \& Reynolds, S. P. 1994, ApJ, 437, 705

Nakayama, M., \& Masai, K. 2001, ApJ, 375, 328

Pye, J. P., Becker, R. H., Seward, F. D., \& Thomas, N. 1984, MNRAS, 207, 649

Radhakrishnan, V., Goss, W. M., Murray, J. D., \& Brooks, J. W. 1972, ApJS, 24,49

Serlemitsos, P. J., et al. 2007, PASJ, 59, 9

Smith, R. K., \& Brickhouse, N. S. 2002, Physics Underlying the ATOMDB (Cambridge, MA: Chandra X-Ray Center), http://cxc.harvard.edu/atomdb/physics/plasma/plasma.html

Smith, R. K., Brickhouse, N. S., Liedahl, D. A., \& Raymond, J. C. 2001, ApJ, 556, L91

Uchiyama, H., et al. 2009, PASJ, 61, 9

Wargelin, B. J., Beiersdorfer, P., Neill, P. A., Olson, R. E., \& Scofield, J. H. 2005, ApJ, 634, 687

Yamaguchi, H., Ozawa, M., Koyama, K., Masai, K., Hiraga, J. S., Ozaki, M., \& Yonetoku, D. 2009, ApJ, 705, 6 